\title{
Continuous Finite Element Methods of Molecular Dynamics Simulations
}

\author{
Qiong Tang, ${ }^{1}$ Luohua Liu, ${ }^{1}$ and Yujun Zheng ${ }^{2}$ \\ ${ }^{1}$ College of Science, Hunan University of Technology, Zhuzhou, Hunan 412007, China \\ ${ }^{2}$ Department of Mathematics and Computational Science, Hunan University of Science and Engineering, \\ Yongzhou, Hunan 425100, China
}

Correspondence should be addressed to Qiong Tang; zzgxysx@163.com

Received 13 February 2015; Accepted 26 July 2015

Academic Editor: Ahmed Rachid

Copyright (C) 2015 Qiong Tang et al. This is an open access article distributed under the Creative Commons Attribution License, which permits unrestricted use, distribution, and reproduction in any medium, provided the original work is properly cited.

\begin{abstract}
Molecular dynamics simulations are necessary to perform very long integration times. In this paper, we discuss continuous finite element methods for molecular dynamics simulation problems. Our numerical results about $A B$ diatomic molecular system and $A_{2} B$ triatomic molecules show that linear finite element and quadratic finite element methods can better preserve the motion characteristics of molecular dynamics, that is, properties of energy conservation and long-term stability. So finite element method is also a reliable method to simulate long-time classical trajectory of molecular systems.
\end{abstract}

\section{Introduction}

Molecular dynamics (MD) simulations can provide detailed information on molecular fluctuations and conformational changes and are used to investigate the thermodynamics and structure of chemical and biological molecules. Constructing efficient algorithms suitable for MD applications is an important task. Because of the very rapid oscillatory motions characteristic of $\mathrm{MD}$, very small time steps of about a femtosecond $\left(10^{-15}\right)$ are typically used in MD. This means that, in order to get estimates for parameters of interest from simulation, it is necessary to perform very long integration times for MD simulations. The total energy $H$, obtained by summing the kinetic and potential contributions, is a conserved quantity for the molecular system. Many numerical methods have been developed but the most effective for use in MD simulations should have superior long-term stability properties and energy conservation and permit a large integration time step [1-6]. It is important to construct discrete algorithms which preserve these basic properties.

Currently symplectic algorithm is the main choice in the long-time calculation to the MD. An important feature is that the molecular systems always remain Hamiltonian dynamical models. The Hamiltonian system has a symplectic structure with strong geometric properties of a dynamical system and conserves the total energy $H(q, p)$ in which the phase-space points $(q, p)$ are allowed on the constant energy hypersurface satisfying $H(q, p)=E$. Ruth, Feng, Sanz-Serna, and Marsden et al. constructed the symplectic algorithm to solve the Hamiltonian system, including the 4-stage, 4th-order explicit symplectic scheme (4SS) to the separable Hamiltonian system and 2-stage, 4th-order implicit symplectic Runge-Kutta scheme (4SRK) [4, 7-13]. It is a difference method that preserves the structure of the system. However, most numerical methods cannot maintain the two properties: Symplectic and energy conservation simultaneously in general according to Ge-Marsde theorem [14]. Symplectic algorithm can preserve symplectic properties, but it can only obtain approximate energy conservation for nonlinear Hamiltonian system. However, the energy conservation is very important to the MD system. If not, $(p, q)$ do not stay near the energy surface very soon leading to unrealistic averages $[4-6,15]$.

Applying continuous finite element methods for Hamiltonian systems, there are two important advantages where finite element methods can preserve energy conservation and high accuracy approximation to symplectic structure. Zhong and Chen et al. [16-18] got good results. Based on the 
importance of energy conservation and long time to the MD system, in this paper, we first use continuous finite element methods to solve MD simulation problems. In numerical experiments of $A B$ diatomic molecular and $A_{2} B$ triatomic molecules, the linear element and quadratic element methods can better preserve the motion characteristics of molecular system: conservation of energy and long-term stability properties, that reached the microscopic reaction need to be considered time. It is a reliable method for long-time classical trajectory simulations of MD.

\section{Continuous Finite Element Methods for Hamiltonian Systems}

Consider the first-order ordinary differential equation with initial value in the interval $J=[0, T]$ :

$$
\begin{aligned}
\frac{d u}{d t} & =u^{\prime}=f(t, u), \\
u(0) & =u_{0} .
\end{aligned}
$$

Let $J^{h}: t_{0}=0<t_{1}<t_{2}<\cdots<t_{N}=T$ be a partition of $J$, with $I_{j}=\left(t_{j-1}, t_{j}\right), h_{j}=t_{j}-t_{j-1}$. Consider $h=\max h_{j}$, $h_{j} \geq C h, 1 \leq j \leq N$. The constant $C$ is independent of $j$ and $h$. Define the $m$ th degree continuous finite element space:

$$
S^{h}=\left\{w|w \in C(J), w|_{I_{j}} \in P_{m}\right\},
$$

where $P_{m}$ is a set of $m$ th degree polynomials. In cell $I_{j}, m$ th degree polynomial has $m+1$ parameters. As it is an initial value problem, and we already knew the initial value $U\left(t_{j-1}\right)$ in $I_{j}$, it has only $m$ degrees of freedom. Defining $m$ th degree continuous finite element $U \in S^{h}$ satisfies $[19,20]$

$$
\begin{aligned}
\int_{I_{j}}\left(U^{\prime}-f(t, U)\right) v d t & =0, \quad v \in P_{m-1}, \\
U(0) & =u_{0} .
\end{aligned}
$$

That is, in cell $I_{j}$, it is orthogonal to arbitrary $P_{m-1}$. Taking $v \in S^{h}$, then its derivate $v^{\prime} \in P_{m-1}$. In practical computation, take $v=\left(t-t_{j-1}\right)^{i}, i=0,1,2, \ldots, m-1$.

Lemma 1 (see [20]). The mth degree continuous finite element of ordinary differential equation has superconvergence in cell node $t_{j}$ :

$$
(u-U)\left(t_{j}\right)=O\left(h^{2 m}\right)\|u\|_{m+1, \infty} .
$$

We take finite dimension autonomous Hamiltonian $H(p, q)$ canonical systems as

$$
\begin{aligned}
& \frac{d p}{d t}=-\frac{\partial H}{\partial q} \\
& \frac{d q}{d t}=\frac{\partial H}{\partial p}
\end{aligned}
$$

where $p=\left(p_{1}, p_{2}, \ldots, p_{n}\right)^{T}, q=\left(q_{1}, q_{2}, \ldots, q_{n}\right)^{T}$, and matrix transpose is defined by “ $T$." In applications to $\mathrm{MD}, q$, $p$, and $H$ represent the $n$ particles positions, the canonical momentum, and the total energy of the systems, respectively.

Let $z=[p, q]^{T} ;(5)$ can be written as

$$
\frac{d z}{d t}=z^{\prime}=J^{-1} H_{z}=f(z)
$$

where $J=\left[\begin{array}{cc}0 & I_{n} \\ -I_{n} & 0\end{array}\right], I_{n}$ is the $n$ order identity matrix, and $H_{z}=$ $\left[\begin{array}{l}H_{p} \\ H_{q}\end{array}\right]$.

Define (6)'s $m$ th degree continuous finite element $Z=$ $[P, Q]^{T}$ of $z$; it satisfies orthogonal relation:

$$
\begin{aligned}
\int_{I_{j}}\left(Z^{\prime}+J H_{z}\right) v^{\prime} d t & =0, \\
Z(0) & =z_{0} .
\end{aligned}
$$

Taking $v=[Q, P]^{T}$, we obtain

$$
\begin{aligned}
\int_{I_{j}}\left(P^{\prime}+H_{q}(P, Q)\right) Q^{\prime} d t & =0, \\
P(0) & =p_{0}, \\
\int_{I_{j}}\left(Q^{\prime}-H_{p}(P, Q)\right) P^{\prime} d t & =0, \\
Q(0) & =q_{0} .
\end{aligned}
$$

By subtracting (9) from (8),

$$
\int_{I_{j}}\left(H_{p} P^{\prime}+H_{q} Q^{\prime}\right) d t=\int_{I_{j}} \frac{d}{d t} H(P, Q) d t=0 .
$$

Hence, in each node $t_{j}$, we prove that

$$
\begin{aligned}
H\left(P\left(t_{j}\right), Q\left(t_{j}\right)\right) & =H\left(P\left(t_{j-1}\right), Q\left(t_{j-1}\right)\right)=\cdots \\
& =H(P(0), Q(0))=H\left(p_{0}, q_{0}\right) .
\end{aligned}
$$

Then we can prove the following.

Lemma 2 (see [16]). Applying arbitrary degree continuous finite element to solve Hamilton equation, it preserves energy conservation; that is, $H\left(P\left(t_{j}\right), Q\left(t_{j}\right)\right)=H\left(p_{0}, q_{0}\right)$.

Using continuous finite element methods to solve nonautonomous Hamiltonian $H(p, q, t)$ systems, from (7), we can obtain

$$
\begin{aligned}
\int_{I_{j}}\left(P^{\prime}+H_{q}(P, Q, t)\right) Q^{\prime} d t & =0, \\
P(0) & =p_{0}, \\
\int_{I_{j}}\left(Q^{\prime}-H_{p}(P, Q, t)\right) P^{\prime} d t & =0, \\
Q(0) & =q_{0} .
\end{aligned}
$$


By subtracting (13) from (12),

$$
\begin{aligned}
\int_{I_{j}} & \left(H_{p}(P, Q, t) P^{\prime}+H_{q}(P, Q, t) Q^{\prime}\right) d t \\
= & \int_{I_{j}}\left(\frac{d}{d t} H(P, Q, t)-H_{t}(P, Q, t)\right) d t=0 .
\end{aligned}
$$

It is a nonautonomous Hamiltonian system; in general $\int_{I_{j}} H_{t}(p, q, t) d t \neq 0$ for every cell $I_{J}$. Hence, in node $t_{j}$,

$$
H\left(P\left(t_{j}\right), Q\left(t_{j}\right), t_{j}\right) \neq H\left(p_{0}, q_{0}, t_{0}\right) .
$$

Theorem 3. The continuous finite element methods cannot preserve the energy to nonautonomous Hamiltonian systems.

Theorem 3 verifies that the nonautonomous Hamiltonian systems $H(p, q, t)$ do not have the energy conservation, which coincides with the actual case.

Definition 4 (see [21]). A transformation $z \rightarrow \widehat{z}$ of $R^{2 n}$ is called canonical, or symplectic, if it is a local diffeomorphism whose Jacobian $\partial \widehat{z} / \partial z$ is everywhere symplectic; that is,

$$
\left(\frac{\partial \widehat{z}}{\partial z}\right)^{T} J \frac{\partial \widehat{z}}{\partial z}=J
$$

Utilizing Legendre orthogonal polynomial and Lemmas 1 and 2 , we can prove the following.

Lemma 5 (see [17]). The linear finite element for nonlinear Hamiltonian systems is an approximately symplectic algorithm which has the accuracy of third order to Hamiltonian systems symplectic structure; that is, $\left(\partial Z_{j+1} / \partial Z_{j}\right)^{T} J\left(\partial Z_{j+1} / \partial Z_{j}\right)=J+$ $\mathrm{O}\left(h^{3}\right)$. It also possesses second-order accuracy and preserves the energy.

Lemma 6 (see [17]). The quadratic finite element for nonlinear Hamiltonian systems is an approximately symplectic algorithm which has the accuracy of fifth order to Hamiltonian systems symplectic structure; that is, $\left(\partial Z_{j+1} / \partial Z_{j}\right)^{T} J\left(\partial Z_{j+1} / \partial Z_{j}\right)=J+$ $O\left(h^{5}\right)$. It also possesses fourth-order accuracy and preserves the energy.

The specific calculation format of the linear continuous finite element $(1 \mathrm{FE}) Z=((1-x) / 2) Z_{j-1}+((1+x) / 2) Z_{j}$, $x \in[-1,1]$, is as follows:

$$
Z_{j}-Z_{j-1}=\frac{h_{j}}{2} J \int_{-1}^{1} H_{z}(Z) d x, \quad j=0,1, \ldots, N-1 .
$$

The specific calculation format of the quadratic continuous finite element $(2 \mathrm{FE}) Z=\left(\left(x^{2}-x\right) / 2\right) Z_{j-1}+\left(1-x^{2}\right) Z_{j-1 / 2}+$ $\left(\left(x^{2}+x\right) / 2\right) Z_{j}, Z_{j-1 / 2}=Z\left(\left(t_{j-1}+t_{j}\right) / 2\right), x \in[-1,1]$, is as follows:

$$
\begin{aligned}
Z_{j}-Z_{j-1} & =\frac{h_{j}}{2} J \int_{-1}^{1} H_{z}(Z) d x \\
\frac{5}{6} Z_{j}-\frac{2}{3} Z_{j-1 / 2}-\frac{1}{6} Z_{j-1} & =\frac{h_{j}}{4} J \int_{-1}^{1} H_{z}(Z)(x+1) d x
\end{aligned}
$$

We utilize high accuracy numerical integration such as at least $m+1$ points of the Gaussian quadrature formula to the $m$ th degree continuous finite element at the right of (17) or (18). From above equation set, we can solve $Z_{j}$ on cell $I_{j}=\left(t_{j-1}, t_{j}\right)$, and then we can get the value $Z_{j}, j=1, \ldots, N$.

\section{Numerical Experiments}

3.1. Diatomic Molecular System. Consider the classical motion of $A B$ diatomic molecule in the electronic potential, where the mass of atom $A$ is $m_{1}$ and that of atom $B$ is $m_{2}$. The coordinates of $A$ and $B$ are $x_{1}$ and $x_{2}$, respectively. $q=R=x_{1}+x_{2}$ is the canonical coordinate; $m=\left(m_{1}+m_{2}\right) /\left(m_{1} m_{2}\right)$ is the canonical mass. Then $p=m q_{t}$ and $U=p^{2} /(2 m)$ are the momentum and canonical kinetics, respectively. Morse potentials have been used to describe vibrational levels of diatomic molecules. We take the Morse potential as $V(q)=D\left(e^{-2 \alpha\left(q-q_{0}\right)}-e^{-\alpha\left(q-q_{0}\right)}\right)$ [6]. In each case, the parameters $D, \alpha$, and $q_{0}$ of the corresponding Morse potential are specified. The total energy of the $A B$ diatomic molecule system is

$$
H(p, q)=U(p)+V(q)=\frac{1}{2 m} p^{2}+V(q)
$$

And the Hamiltonian equation is

$$
\begin{aligned}
& \frac{d p}{d t}=-\frac{\partial H}{\partial q}=-\frac{\partial V(q)}{\partial q} \\
& \frac{d q}{d t}=\frac{\partial H}{\partial p}=\frac{\partial U(p)}{\partial p}
\end{aligned}
$$

Since it is a separated Hamiltonian system, symplectic scheme can be used to solve this system [6]. We consider 2-stage, 4 thorder implicit symplectic Runge-Kutta scheme (4SRK):

$$
\begin{aligned}
Y_{1} & =z^{n}+h\left(\frac{1}{4} f\left(Y_{1}\right)+\left(\frac{1}{4}-\frac{\sqrt{3}}{6}\right) f\left(Y_{2}\right)\right), \\
Y_{2} & =z^{n}+h\left(\left(\frac{1}{4}+\frac{\sqrt{3}}{6}\right) f\left(Y_{1}\right)+\frac{1}{4} f\left(Y_{2}\right)\right), \\
z^{n+1} & =z^{n}+\frac{h}{2}\left(f\left(Y_{1}\right)+f\left(Y_{2}\right)\right) .
\end{aligned}
$$

The 4-stage, 4th-order explicit symplectic difference scheme (4SS) is

$$
\begin{aligned}
H(p, q) & =T(p)+V(q), \\
\alpha & =\left(2-2^{1 / 3}\right)^{-1}, \\
\beta & =1-2 \alpha, \\
p_{1} & =p^{n}-c_{1} h\left(\frac{\partial V(q)}{\partial q}\right)_{q^{n}},
\end{aligned}
$$




$$
\begin{aligned}
q_{1} & =q^{n}+d_{1} h\left(\frac{\partial T(p)}{\partial p}\right)_{p_{1}}, \\
p_{2} & =p^{n}-c_{2} h\left(\frac{\partial V(q)}{\partial q}\right)_{q_{1}}, \\
q_{2} & =q^{n}+d_{2} h\left(\frac{\partial T(p)}{\partial p}\right)_{p_{2}}, \\
p_{3} & =p^{n}-c_{3} h\left(\frac{\partial V(q)}{\partial q}\right)_{q_{2}}, \\
q_{3} & =q^{n}+d_{3} h\left(\frac{\partial T(p)}{\partial p}\right)_{p_{3}}, \\
p^{n+1} & =p^{n}-c_{4} h\left(\frac{\partial V(q)}{\partial q}\right)_{q_{3}}, \\
q_{n+1} & =q^{n}+d_{4} h\left(\frac{\partial T(p)}{\partial p}\right)_{p^{n+1}},
\end{aligned}
$$

where $c_{1}=c_{4}=\alpha / 2, c_{2}=c_{3}=(\alpha+\beta) / 2, d_{1}=d_{2}=\alpha, d_{3}=$ $\beta$, and $d_{4}=0$.

Take the potential parameters $D=88575.905 \mathrm{~cm}^{-1}, \alpha=$ $2.3425473 \AA^{-1}, m_{1}=22036$ a.u., $m_{2}=29376$ a.u., $m=\left(m_{1} *\right.$ $\left.m_{2}\right) /\left(m_{1}+m_{2}\right)$, and $q_{0}=1.128323 \AA$ to the CO molecule [5]. Initial condition $q(0)=q_{0}, p(0)=\sqrt{2 \mathrm{MD}}-0.01$, stepsize $h=$ 3 , computing $K=10^{8}$ step, and integral interval $T=[0,3 *$ $10^{8}$ ]. Respectively, we utilize $2 \mathrm{FE}, 4 \mathrm{SRK}$, and 4 SS to compute the energy error; two atoms oscillate versus time and $(q, p)$ classical trajectories of the CO diatomic systems as follows (Figures 1-6).

Take the potential parameters $D=51443.117 \mathrm{~cm}^{-1}$, $\alpha=24.251821 \mathrm{~nm}^{-1}, m_{1}=25704$ a.u., $m_{2}=m_{1}, m=$ $m_{1} / 2$, and $q_{0}=0.12203 \mathrm{~nm}$ to the $N_{2}$ molecule [5]. Initial condition $q(0)=q_{0}, p(0)=\sqrt{2 \mathrm{MD}}-0.01$, stepsize $h=3$, computing $K=10^{8}$ step, and integral interval $T=[0,3 *$ $10^{8}$ ]. Respectively, we utilize $2 \mathrm{FE}, 4 \mathrm{SRK}$, and 4 SS to compute the energy error and $(q, p)$ classical trajectories of the $\mathrm{N}_{2}$ diatomic systems as follows (Figures 7-9).

Figures 1, 3, 5, 7, 8, and 9 show the evolution of the energy and the phase trajectories of $\mathrm{CO}, \mathrm{N}_{2}$ molecule versus time. Figures 2, 4, and 6 indicate that two atoms oscillate in the evolution process, respectively, utilizing $2 \mathrm{FE}, 4 \mathrm{SRK}$, and 4SS. It is observed from Figures 1-9 that the numerical results computed by $2 \mathrm{FE}$ are in agreement with the theoretical analysis, the phase trajectories in phase space are steady, and two atoms oscillate periodically in the evolution process (Figures 1, 2, and 7) which indicates that 2FE can keep approximate high accuracy of symplectic structure just as the symplectic difference method (Figures 3, 4, 5, 6, 8, and 9) which preserves the structure of phase space in a long time $\left(T=\left[0,3 * 10^{8}\right]\right)$. The energy error computed by $2 \mathrm{FE}$ is only $10^{-15}$ (Figures 1 and 7) which indicates conservation of energy over long time intervals $T=3 * 10^{8}$ under a large stepsize
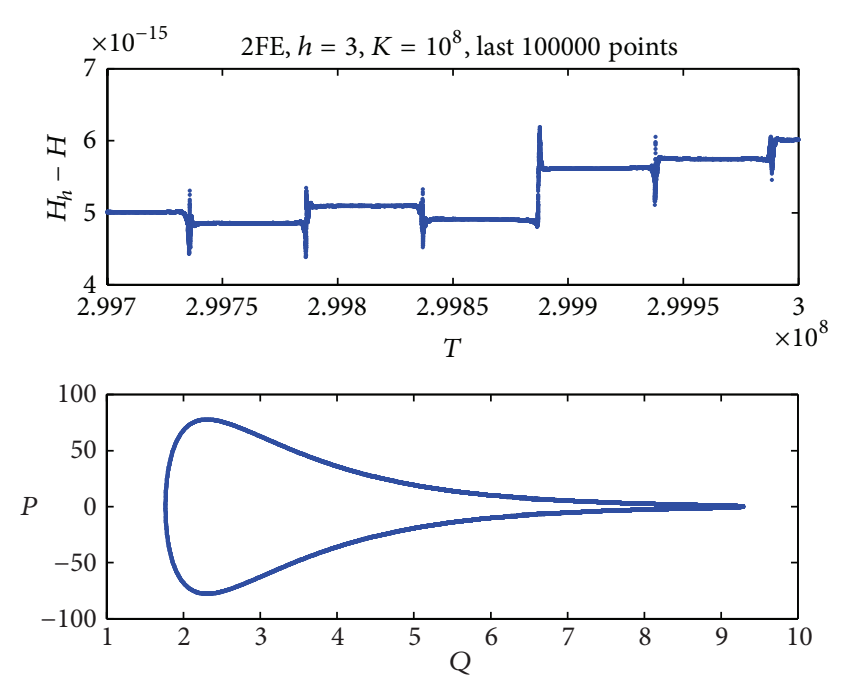

Figure 1: 2FE, computing $K=10^{8}$ step, the final 100000 points' corresponding energy error curve and phase trajectories of $\mathrm{CO}$ molecule in the phase space $(q, p)$.
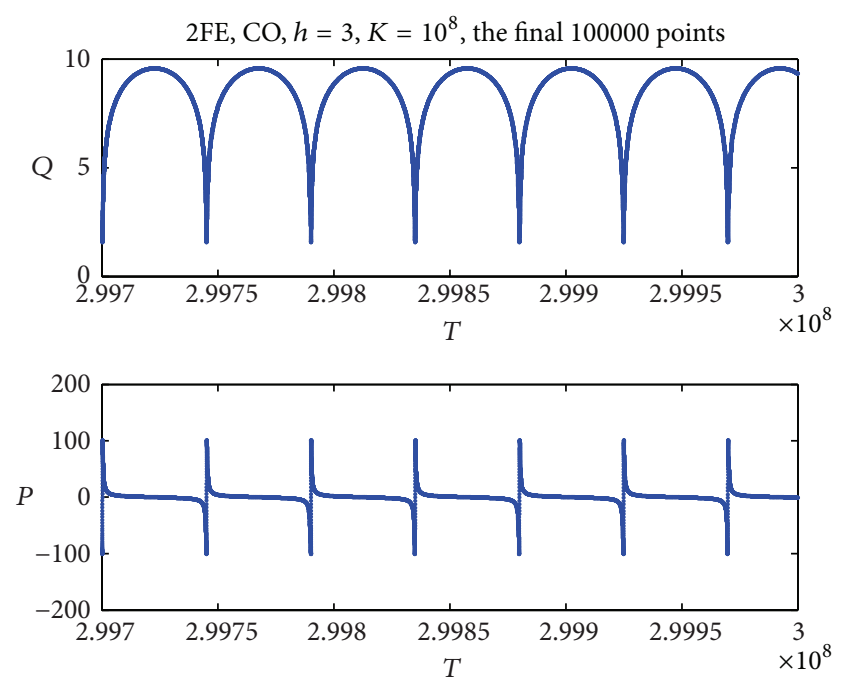

FIgURE 2: 2FE, computing $K=10^{8}$ step, the evolution of the final 100000 points $p(t), q(t)$.

$h=3$, but the energy deviation is comparatively larger by symplectic difference scheme, energy error up to $10^{-9}$ (4SRK, Figures 3 and 8 ) and $10^{-7}$ (4SS, Figures 5 and 9), which is only approximately conservative.

3.2. $A_{2} B$ Triatomic Molecules. Basing Banerjee and Adams constructed a transformation method; we consider the motion of $\mathrm{A}_{2} \mathrm{~B}$ triatomic molecule such as water $\left(\mathrm{H}_{2} \mathrm{O}\right)$ within the $C_{2 v}$ symmetry; take the Cartesian coordinate system $y \mathrm{O} z$, with origin at the center of mass $O$, and the $z$ axis is the $C_{2}$ axis; the coordinates of the two $A$ atoms and $B$ atom are $A\left(y_{1}, z_{1}\right), A\left(y_{2}, z_{2}\right)$, and $B\left(y_{3}, z_{3}\right)$. The generalized 

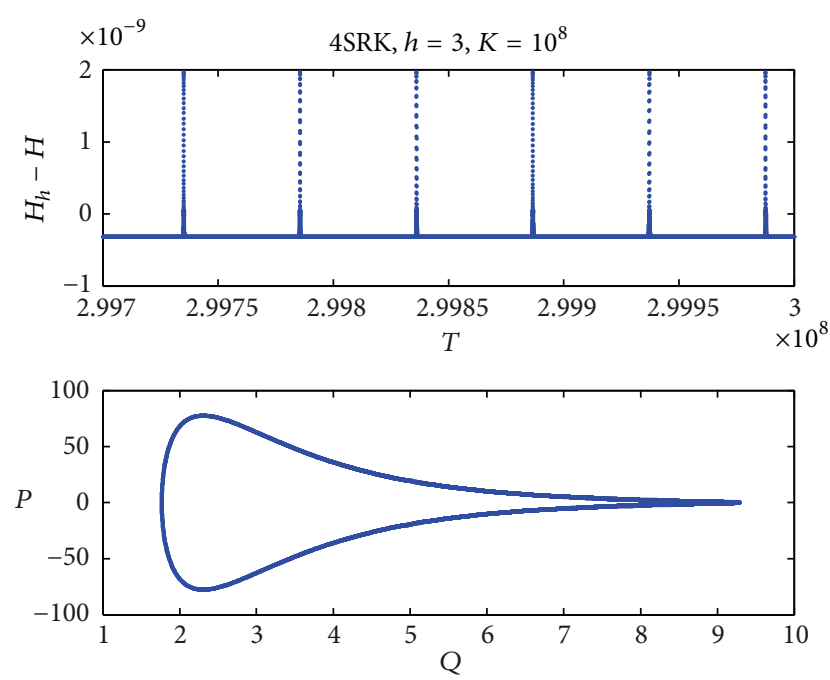

FIgURE 3: 4SRK, computing $K=10^{8}$ step, the final 100000 points' corresponding energy error curve and phase trajectories of $\mathrm{CO}$ molecule in the phase space $(q, p)$.
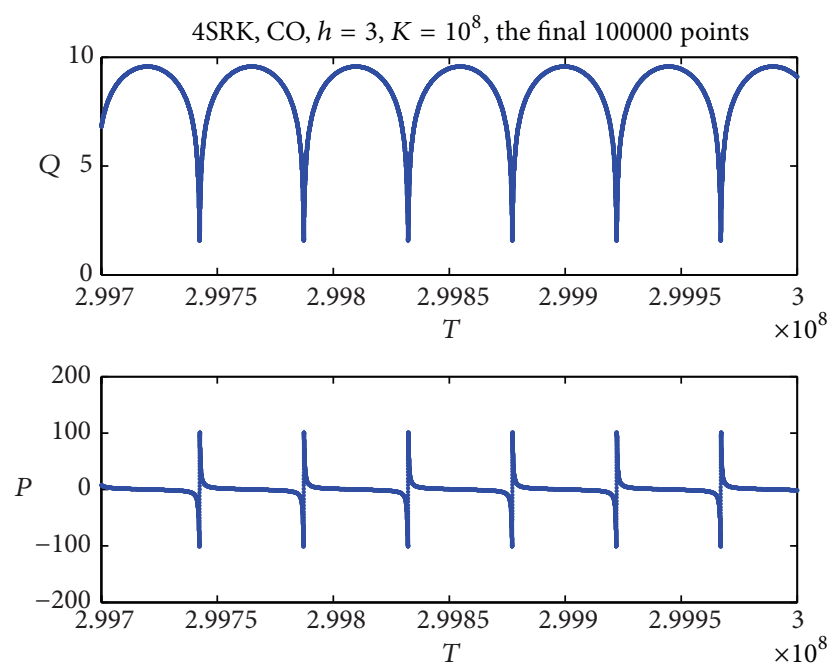

FIGURE 4: 4SRK, computing $K=10^{8}$ step, the evolution of the final 100000 points $p(t), q(t)$.

coordinates $q_{1}=y_{1}-y_{2}, q_{2}=z_{1}-2 z_{3}+z_{2}$, and the generalized momenta $p_{1}=0.25\left(d q_{1} / d t\right), p_{2}=0.5\left(d q_{2} / d t\right)$. Hamiltonian function of $A_{2} B$ type molecule is $H(q, p)=K(p)+V(q)$, where $K(p)=2 p_{1}^{2}+p_{2}^{2}$ is the kinetic energy and $V(q)$ is the potential energy:

$$
\begin{aligned}
& V(q)=5 \pi^{2}\left(D^{2}-5 D+6.5\right)+4 D^{-1} \\
& +0.5 \pi^{2}\left(\left|q_{2}\right|-1.5\right)^{2}+\left|q_{2}\right|^{-1}, \\
& \quad D=\left(q_{1}^{2}+q_{2}^{2}\right)^{1 / 2} .
\end{aligned}
$$
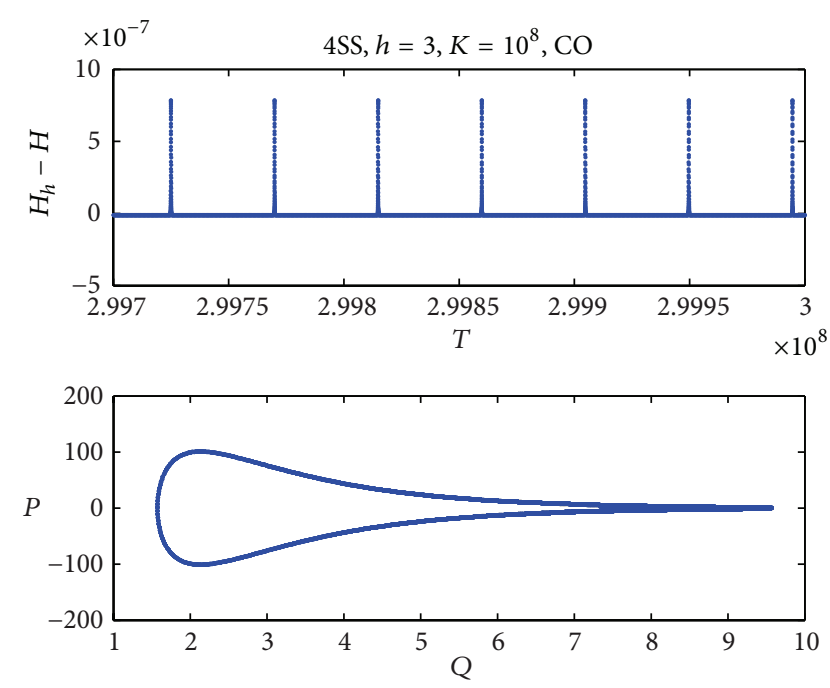

FIgURE 5: 4SS, computing $K=10^{8}$ step, the final 100000 points' corresponding energy error curve and phase trajectories of $\mathrm{CO}$ molecule in the phase space $(q, p)$.
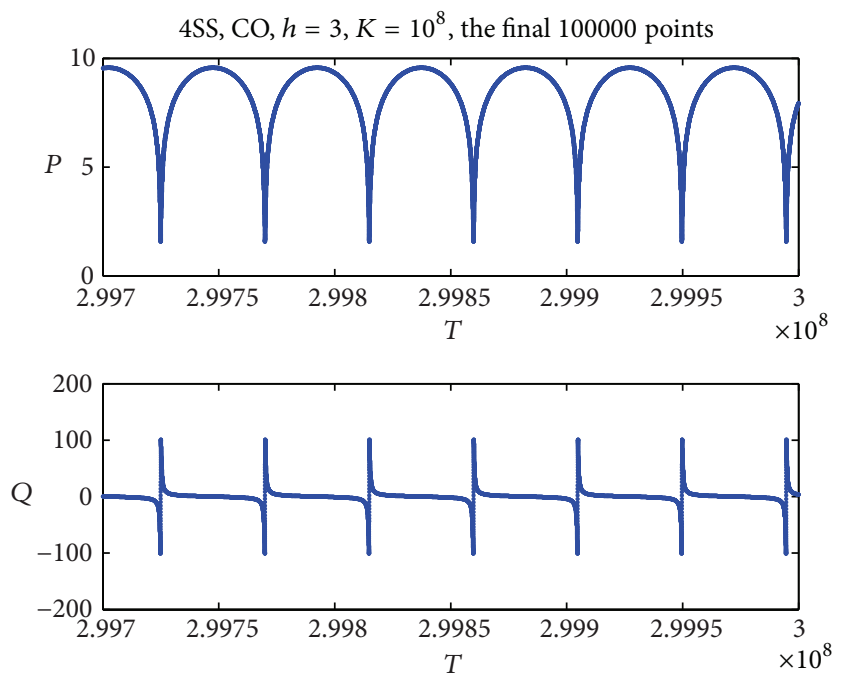

FIGURE 6: 4SS, computing $K=10^{8}$ step, the evolution of the final 100000 points $p(t), q(t)$.

The canonical differential equations are

$$
\begin{aligned}
& \frac{d q_{1}}{d t}=\frac{\partial H}{\partial p_{1}}, \\
& \frac{d q_{2}}{d t}=\frac{\partial H}{\partial p_{2}}, \\
& \frac{d p_{1}}{d t}=-\frac{\partial H}{\partial q_{1}}, \\
& \frac{d p_{2}}{d t}=-\frac{\partial H}{\partial q_{2}} .
\end{aligned}
$$



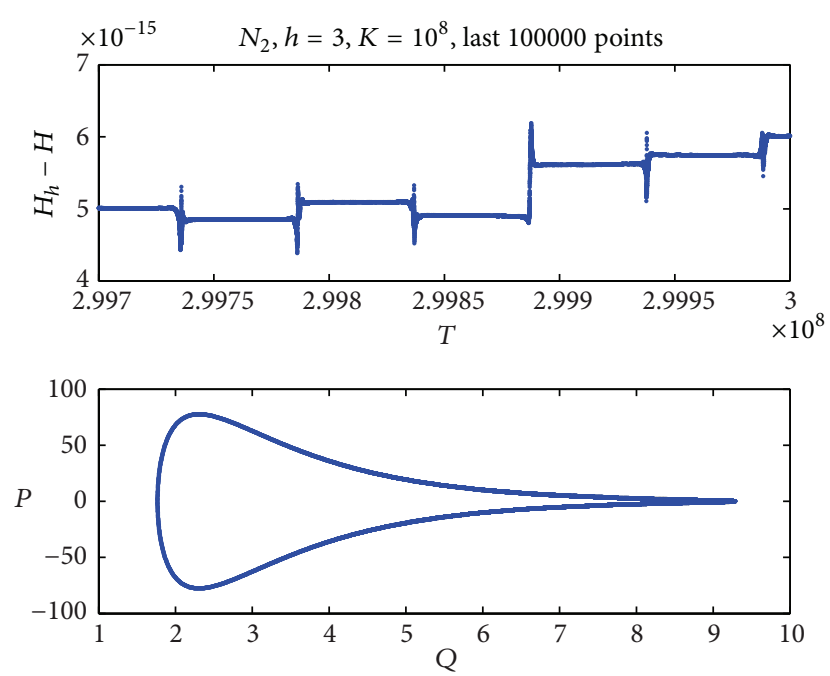

Figure 7: 2FE, computing $K=10^{8}$ step, the final 100000 points' corresponding energy error curve and phase trajectories of $\mathrm{N}_{2}$ molecule in the phase space $(q, p)$.
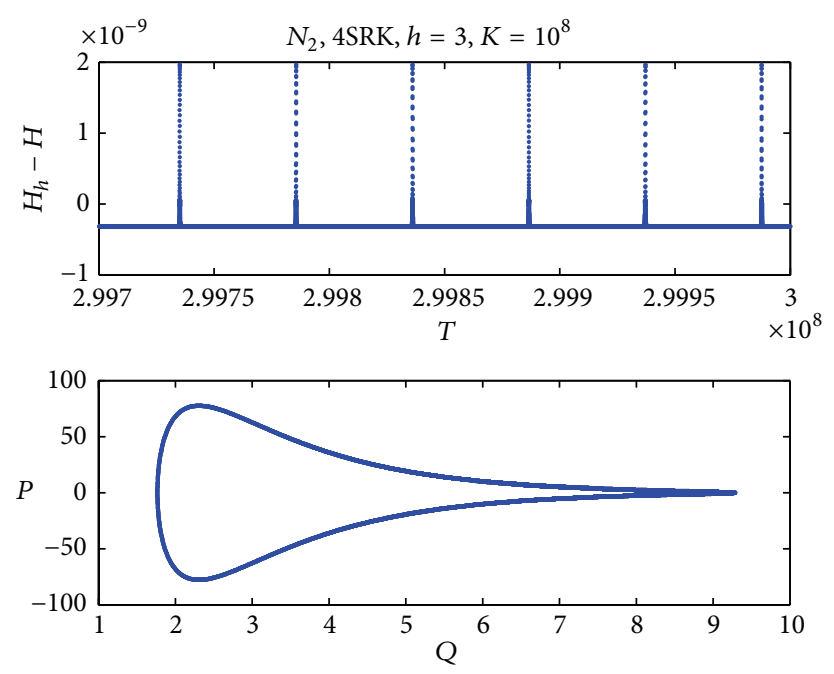

FIgURE 8: 4SRK, computing $K=10^{8}$ step, the final 100000 points' corresponding energy error curve and phase trajectories of $N_{2}$ molecule in the phase space $(q, p)$.

Initial condition: $q_{1}(0)=3, q_{2}(0)=3 / 2, p_{1}(0)=0, p_{2}(0)=0$, and $H(0)=50.1951$. Taking stepsize $h=0.01$, computing $K=10^{8}$ step, integral interval $T=\left[0,10^{6}\right]$.

The second-order symplectic difference scheme (2SS) is

$$
z_{k+1}=z_{k}+h J^{-1} H_{z}\left(\frac{z_{k+1}+z_{k}}{2}\right) \text {. }
$$

We use the linear element method (1FE), general Leapfrog method [1], 2SS, and 4SS to compute the classical trajectories of $A_{2} B$ type molecule in phase spaces and energy error $H_{h}-$ $H$ as follows (Figures 10-13).

It is observed from Figures 10-13 that the numerical results computed by the linear element methods are in agreement with the theoretical analysis; atom $B$ and two $A$ atoms in $A_{2} B$ type molecule vibrate quasi-periodically
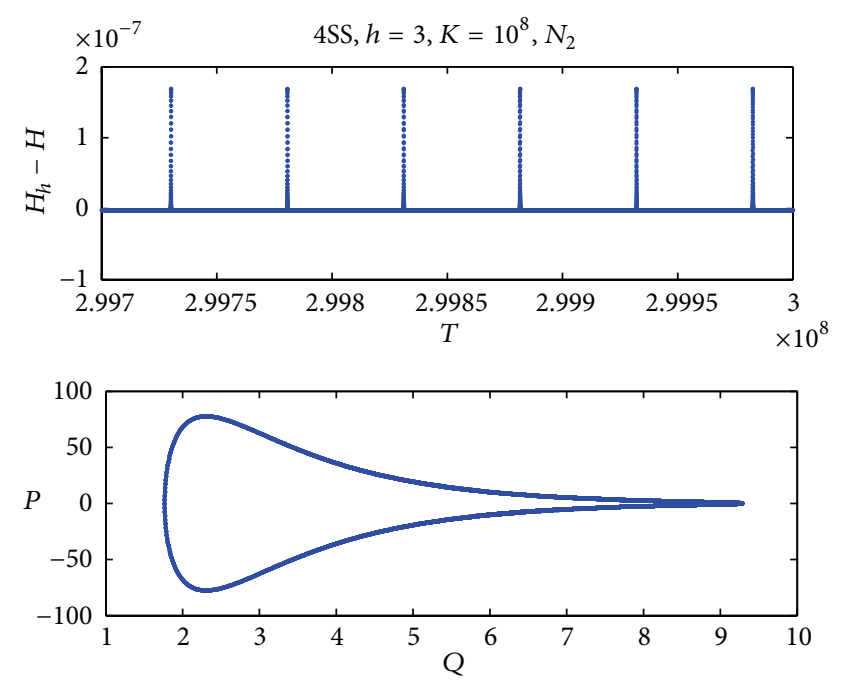

FIgURE 9: 4SS, computing $K=10^{8}$ step, the final 100000 points' corresponding energy error curve and phase trajectories of $\mathrm{N}_{2}$ molecule in the phase space $(q, p)$.
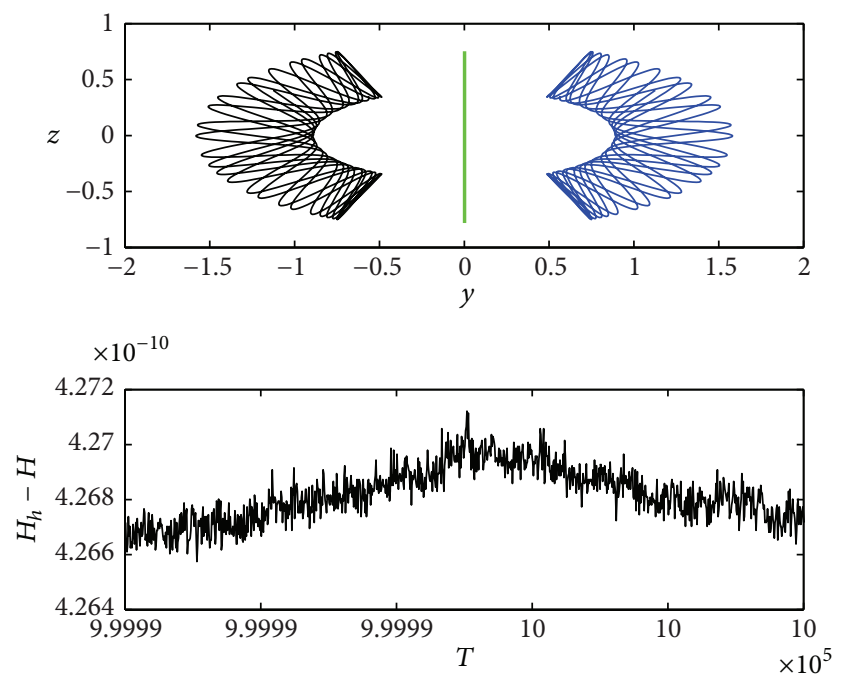

Figure 10: 1FE, computing $K=10^{8}$ step, the final 1000 nodes classical trajectories in phase space and corresponding energy error curve.

and the phase spaces are not squeezed together (Figure 10) which indicates that $1 \mathrm{FE}$ can for a long time preserve the characteristic of the oscillatory motions when $T=1000000$, just as the symplectic difference method (Figures 11 and 12). The energy error computed by the linear element methods is only $10^{-10}$ when $T=1000000$, it reached the microscopic reaction needed to be considered time $T=h * K=10^{6}$, but the energy deviation is comparatively larger by symplectic difference scheme, energy error up to $10^{-1}$ (Leapfrog method, Figure 11), $10^{-2}$ (2SS, Figure 12), and $10^{-3}$ (4SS, Figure 13), which is only approximately conservative. 

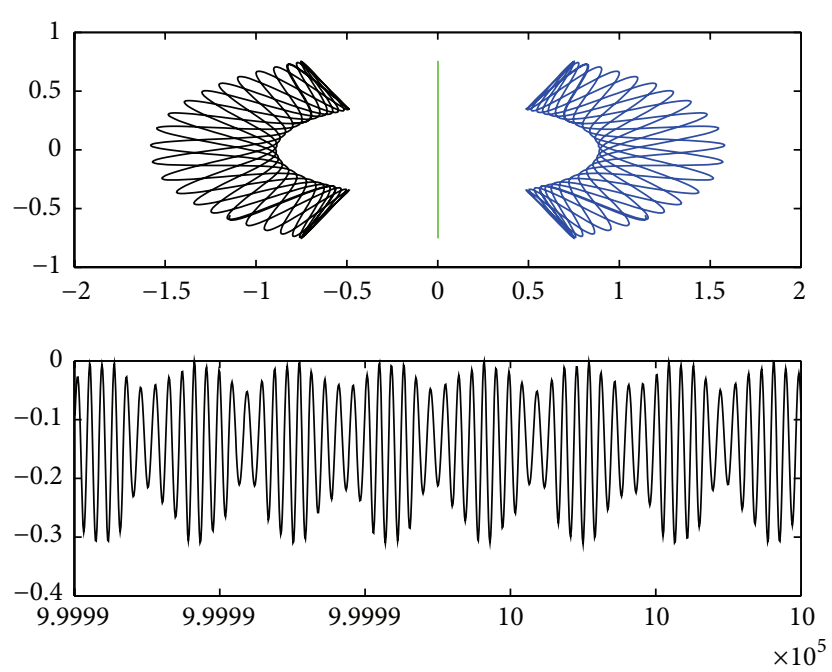

FIgURE 11: General Leapfrog method, computing $K=10^{8}$ step, the final 1000 nodes classical trajectories in phase space and corresponding energy error curve.
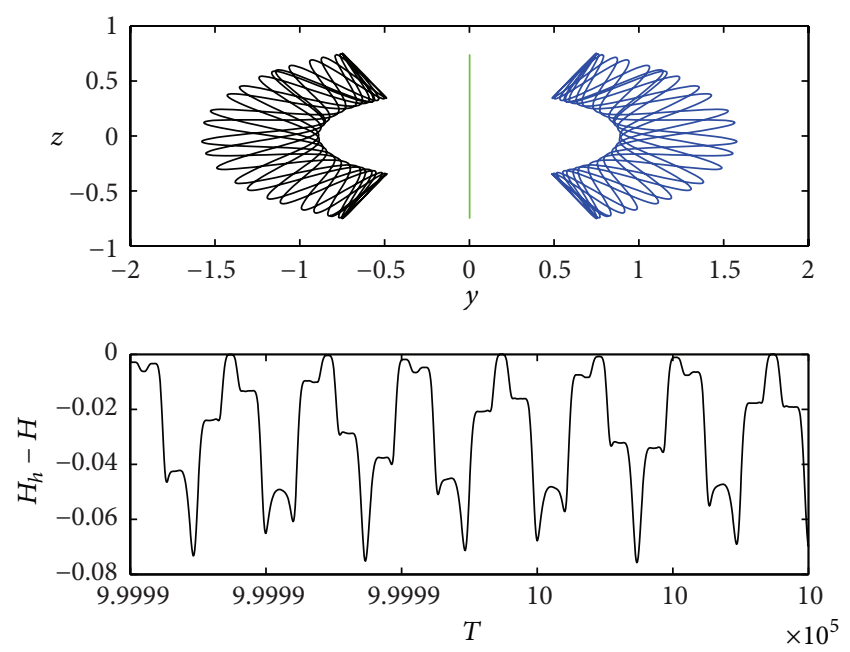

FIGURE 12: 2SS, computing $K=10^{8}$ step, the final 1000 nodes classical trajectories in phase space and corresponding energy error curve.

\section{Conclusion}

The above computations indicate that the finite element methods can preserve less energy drift and are extremely stable over long runs to MD, which leads to an excellent long-time behaviour. These just meet the requirements of MD simulations which have superior long-term stability properties, conservation of energy, and a large integration time step.

\section{Conflict of Interests}

The authors declare that there is no conflict of interests regarding the publication of this paper.
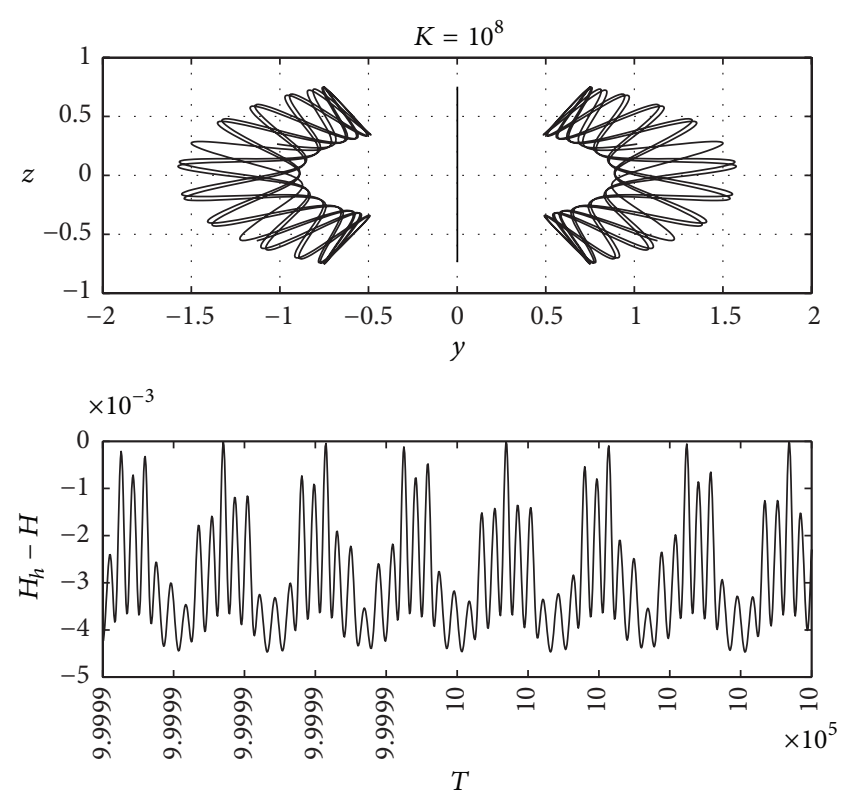

FIgURE 13: 4SS, computing $K=10^{8}$ step, the final 1000 nodes classical trajectories in phase space and corresponding energy error curve.

\section{Acknowledgments}

This research was supported by the National Natural Science Foundation of China (no. 11101136) and aid program for Science and Technology Innovative Research Team in Higher Educational Institutions of Hunan. The authors thank the anonymous reviewer for their useful comments which were helpful to the presentation of this paper.

\section{References}

[1] S. D. Bond, B. J. Leimkuhler, and B. J. Laird, "The nosé-poincaré method for constant temperature molecular dynamics," Journal of Computational Physics, vol. 151, no. 1, pp. 114-134, 1999.

[2] S. D. Bond and B. J. Leimkuhler, "Molecular dynamics and the accuracy of numerically computed averages," Acta Numerica, vol. 16, pp. 1-65, 2007.

[3] J. B. Sturgeon and B. B. Laird, "Symplectic algorithm for constant-pressure molecular dynamics using a Nosé-Poincaré thermostat," Journal of Chemical Physics, vol. 112, no. 8, pp. 3474-3482, 2000

[4] B. Leimkuhler and S. Reich, Simulating Hamiltonian Dynamics, Cambridge University Press, Cambridge, UK, 2004.

[5] E. Ley-Koo, S. Mateos-Cortés, and G. Villa-Torres, "Vibrational-rotational levels and Franck-Condon factors of diatomic molecules via Morse potentials in a box," International Journal of Quantum Chemistry, vol. 58, no. 1, pp. 23-28, 1996.

[6] X.-S. Liu, Y.-Y. Qi, J.-F. He, and P.-Z. Ding, "Recent progress in symplectic algorithms for use in quantum systems," Communications in Computational Physics, vol. 2, no. 1, pp. 1-53, 2007.

[7] K. Feng and M. Z. Qin, Symplectic Geometry Algorithm for Hamiltonian systems, Zhejiang Press of Science and Technology, Hangzhou, China, 2004, (Chinese). 
[8] O. Gonzalez and J. C. Simo, "On the stability of symplectic and energy-momentum algorithms for non-linear Hamiltonian systems with symmetry," Computer Methods in Applied Mechanics and Engineering, vol. 134, no. 3-4, pp. 197-222, 1996.

[9] C. Kane, J. E. Marsden, and M. Ortiz, "Symplectic-energymomentum preserving variational integrators," Journal of Mathematical Physics, vol. 40, no. 7, pp. 3353-3371, 1999.

[10] M. Z. Qin, D. L. Wang, and M. Q. Zhang, "Explicit symplectic difference schemes for separable Hamiltonian system," Journal of Computational Mathematics, vol. 9, no. 3, pp. 211-221, 1991.

[11] S. Reich, "Multi-symplectic Runge-Kutta collocation methods for Hamiltonian wave equations," Journal of Computational Physics, vol. 157, no. 2, pp. 473-499, 2000.

[12] J. M. Sanz-Serna and M. P. Calvo, Numerical Hamiltonian Problems, St Edmundsbury Press, Bury St Edmunds, UK, 1994.

[13] A. M. Stuart and A. R. Humphries, Dynamical Systems and Numerical Analysis, Cambridge University Press, 1998.

[14] G. Zhong and J. E. Marsden, "Lie-poisson hamilton-jacobi theory and lie-poisson integrators," Physics Letters A, vol. 133, no. 3, pp. 134-139, 1988.

[15] E. Hairer, C. Lubich, and G. Wanner, Geometric Numerical Integration: Structure-Preserving Algorithms for Ordinary Differential Equations, vol. 31 of Springer Series in Computational Mathematics, Springer, Berlin, Germany, 2nd edition, 2006.

[16] Q. Tang, C.-m. Chen, and L.-h. Liu, "Energy conservation and symplectic properties of continuous finite element methods for Hamiltonian systems," Applied Mathematics and Computation, vol. 181, no. 2, pp. 1357-1368, 2006.

[17] Q. Tang, C. M. Chen, and L. H. Liu, "Finite element methods for Hamiltonian systems," Mathematica Numerica Sinica, vol. 31, no. 4, pp. 393-406, 2009.

[18] W. X. Zhong and Z. Yao, "Time domain FEM and symplectic conservation," Journal of Mechanical Strength, vol. 27, no. 2, pp. 178-183, 2005.

[19] C. M. Chen and Y. Q. Huang, High Accuracy Theory of Finite Element, Hunan Press of Science and Technology, Changsha, China, 1995 (Chinese).

[20] C. M. Chen, Finite Element Superconvergence Construction Theory, Hunan Press of Science and Technology, Changsha, China, 2001 (Chinese).

[21] K. Feng, Collected Works of Feng Kang, National Defence Industry Press, Beijing, China, 1995. 

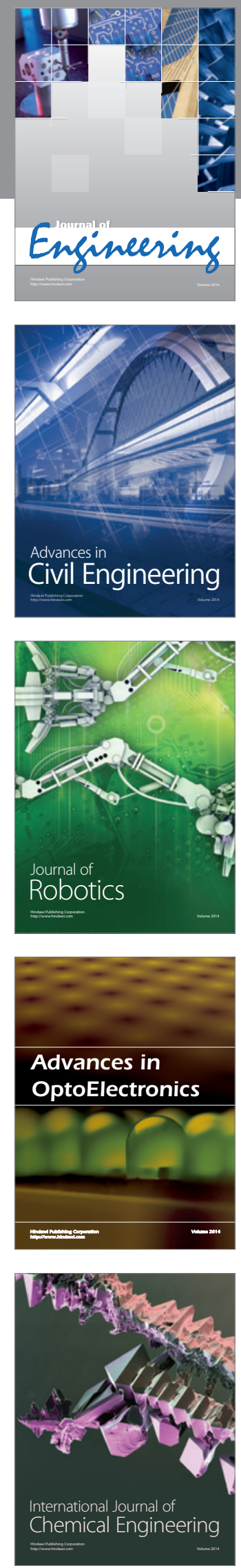

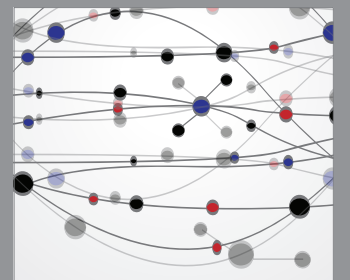

The Scientific World Journal
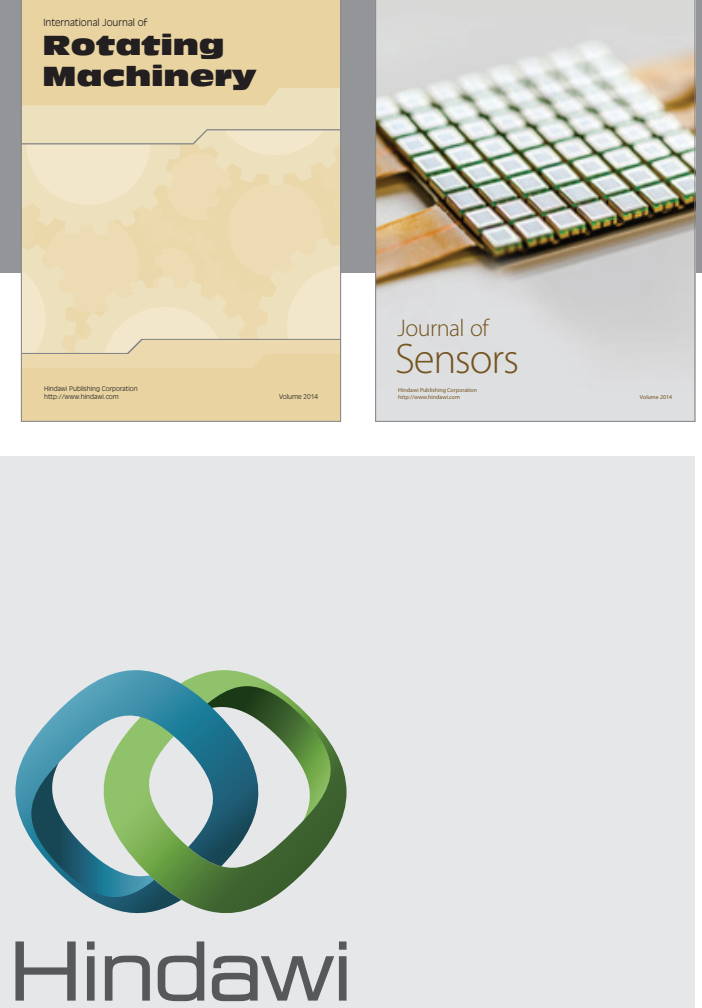

Submit your manuscripts at http://www.hindawi.com
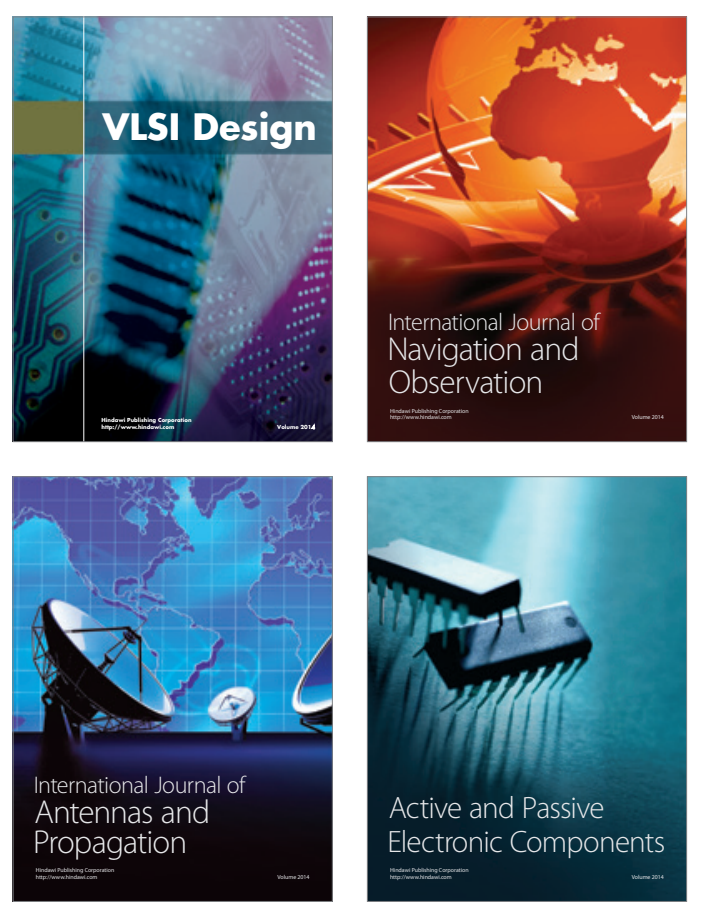
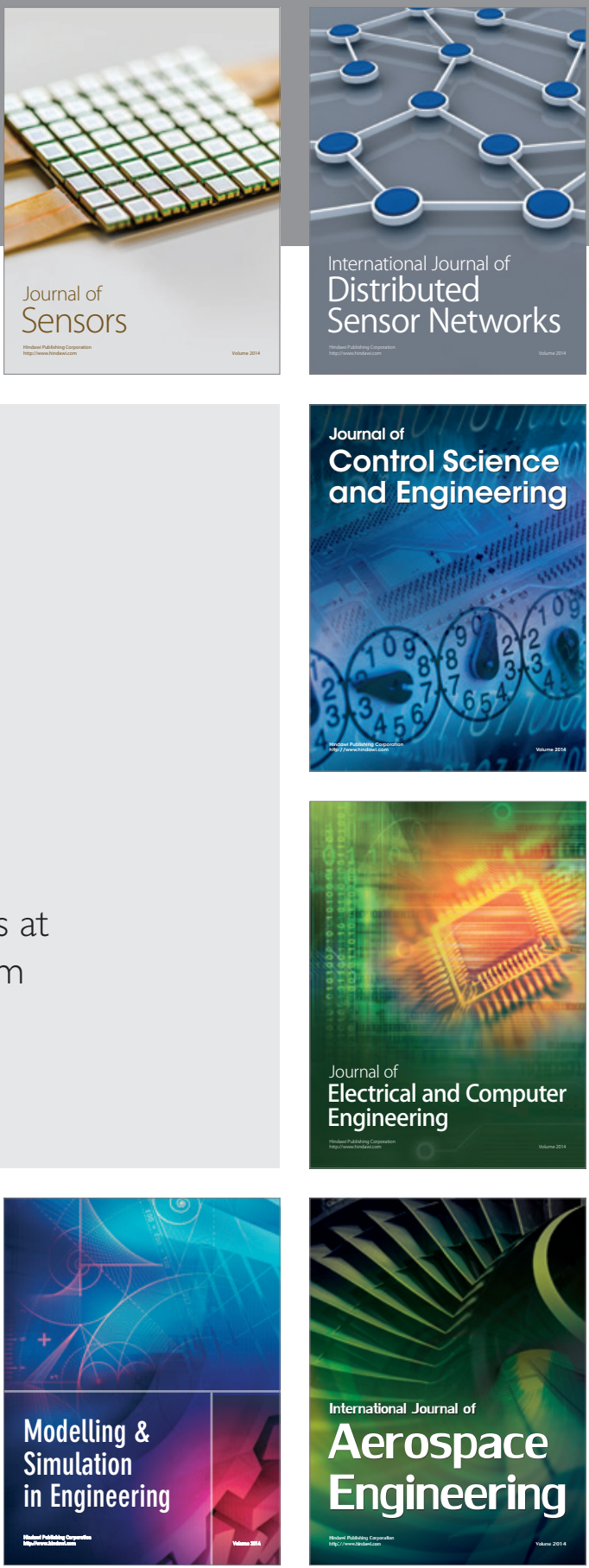

Journal of

Control Science

and Engineering
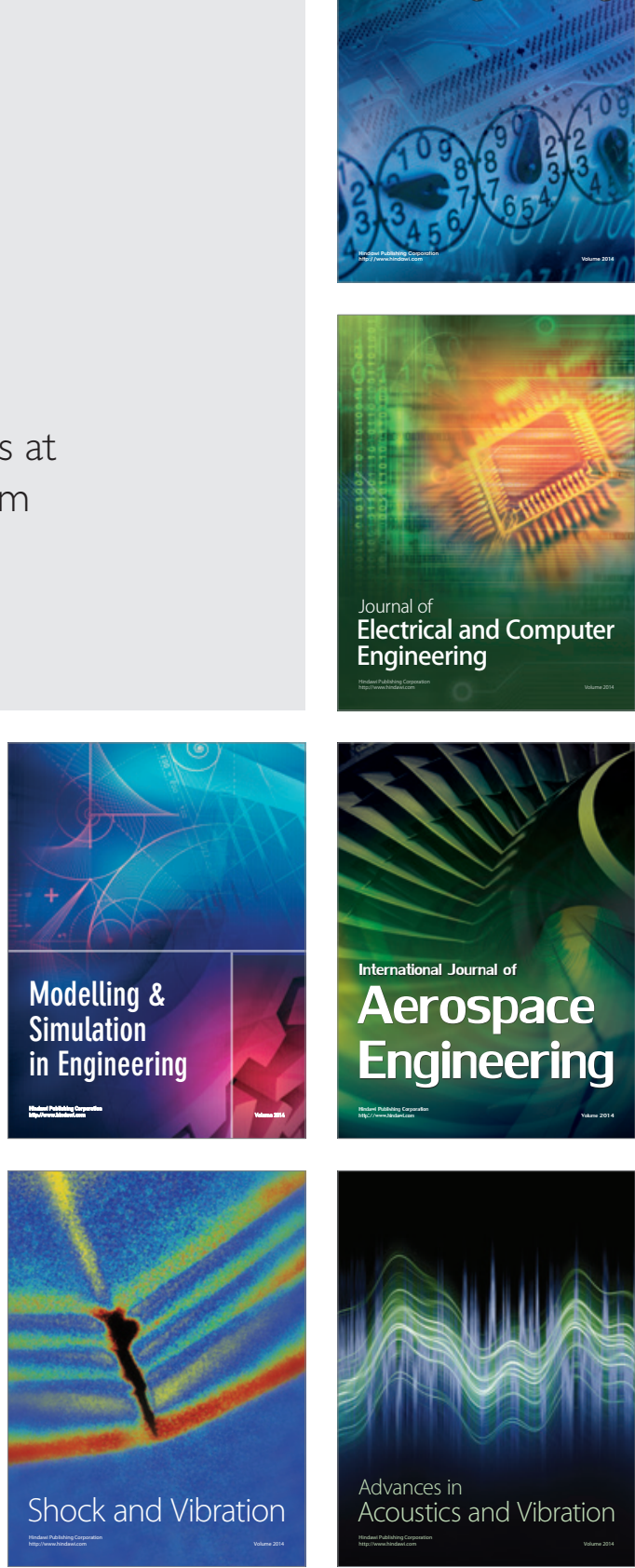